\title{
LA CATALOGNE SUR LE CHEMIN DE L'INDÉPENDANCE ?
}

\author{
Paul Alliès et al.
}

\section{ARPoS | Pôle Sud}

2014/1 - n० 40

pages 5 à 12

\section{ISSN 1262-1676}

Article disponible en ligne à l'adresse:

http://www.cairn.info/revue-pole-sud-2014-1-page-5.htm

Pour citer cet article :

Alliès Paul et al., « La Catalogne sur le chemin de l'indépendance ? »,

Pôle Sud, 2014/1 n 40, p. 5-12.

Distribution électronique Cairn.info pour ARPoS.

(C) ARPoS. Tous droits réservés pour tous pays.

La reproduction ou représentation de cet article, notamment par photocopie, n'est autorisée que dans les limites des conditions générales d'utilisation du site ou, le cas échéant, des conditions générales de la licence souscrite par votre établissement. Toute autre reproduction ou représentation, en tout ou partie, sous quelque forme et de quelque manière que ce soit, est interdite sauf accord préalable et écrit de l'éditeur, en dehors des cas prévus par la législation en vigueur en France. II est précisé que son stockage dans une base de données est également interdit. 


\title{
La Catalogne sur le chemin de l'indépendance ?
}

\author{
Paul Alliès \\ Université de Montpellier I \\ Joan Marcet \\ Université Autonome de Barcelone \\ Institut de Ciències Polítiques i Socials \\ (Traduit de l'espagnol par Jacques Fontaine)
}

La C atalogne, vieille nation européenne, paraît vouée à la confrontation avec l'État auquel elle est rattachée depuis plusieurs siècles. L es conséquences d'une éventuelle sécession de la C atalogne de l'État espagnol transgressent les limites de ces deux territoires et ont des répercussions politiques en Europe. Pôle Sud a souhaité, à travers ce numéro monographique, se pencher sur la réalité politique, économique, sociale et culturelle de la Catalogne, en abordant par là mêmela problématiqueactuelle de sa relation àl'Espagneàlaquelle elle appartient et tout ceci àl'intérieur du contexte po litique européen.

L'expression forte depuis plus de trois ans, c'est le " droit de décider », inassimilable politiquement ou juridiquement au « droit à l'autodétermination » difficilement compatible avec le cadre constitutionnel espagnol, mais également avec celui du droit international. Cependant ce « droit de décider » est parvenu progressivement à s'assimiler à la détermination ou à la volonté d'indépendance par rapport à l'Espagne manifestées par de larges secteurs de la société catalane. En réalité la vie politique catalane, le débat économique, social, culturel, les récents affrontements électoraux, présents et futurs semblent tourner autour, voire même se focaliser quasi exclusivement sur la question de l'hypothèse ou de la crédibilité d'une C atalogne indépendantedel'Espagne.

La formation de l'Espagne en tant qu'État, au cours de la période précédant le libéralisme, de la fin du XV siècle au début du XIXe siècle, est issue d'un processus lent et difficile. Sous l'unité représentée par la monarchie et certaines lois communes, des populations, des structures politiques et des cultures réellement différentes ont survécu. Le changement de dynastie des $\mathrm{H}$ absbourgs aux Bourbons, à l'aube du XVIII ${ }^{e}$ siècle, entraina l'instauration de l'unification juridique ainsi que l'organisation d'un royaume unitaire et fortement centralisé que le libéralisme au XIXe siècle cherche alors à renforcer. En dépit de quelques exceptions ou tentatives avortées au cours du temps, ce modèle unitaire et centralisateur a perduré jusqu'à l'adoption de la Constitution de 1978 qui a fait suite au processus de transition entre la dictature franquiste et la démocratie actuelle, sous la forme politique d'une monarchie parlementaire.

Le modèle instauré par la Constitution actuelle et qui a commencé à se mettreen marche parallelement au processus constituant lui-même, à travers un système complexe de pré-autonomies, a été qualifié d'ouvert et d'ambigu 
étant donné qu'il autorise diverses interprétations, non seulement juridiques ou académiques, mais encore et surtout des lectures politiques différentes. Le nouveau modèle d'organisation de l'État, qui englobe 17 Communautés Autonomes jouissant d'un degré d'autonomie politique remarquable en exerçant toutes des pouvoirs législatifs et exécutifs, non seulement n'est pas une construction abstraite mais prétend apporter une réponse concrète aux problèmesétablis en Espagne en raison del'existence d'un État centralisateur et bureaucratique, imperméable jusqu'al ors aux aspirations de divers secteurs de la population ainsi qu'à l'égard de la réalité multinationale, multiculturelle et plurilinguistique d'un État historiquement conçu comme un État hyper centralisé par les franges les plus conservatrices et réactionnaires.

L'ambigüité propreà ce modèle requérait, au cours des trente-cinq ans et plus de son application, des réal isations concrètes qui permettent un progrès plus en adéquation à l'évolution du modèle propre à l' État et qui répondent davantage aux aspirations nationales de certains de ses territoires, en particulier la Catalogne et le Pays basque, à savoir : depuis la clarification dans la définition et la dètermination des compétences propresà chaque environnement politique jusqu'au perfectionnement du système de financement des Communautés Autonomes; depuis la réforme du Sénat en tant que Chambre de représentation territoriale jusqu'à l'adaptation de l'Administration centrale à la structure nouvelle de l'État décentralisé ou bien encore la participation des Communautés Autonomes aux décisions de ce dernier sur des questions essentielles comme la politiqueà mener dans le cadre del'U nion européenne. La réal ité, c'est qu'aucun des problèmes survenus au cours du processus de développement autonomique n'a pu être résolu, ni aucun des progrès attendusalorsà cetteoccasion seproduireau cours deces dernières années. C eque le milieu académique et le milieu politique lui-même définissaient comme une évolution vers un modèle d'État fédéral, qualifié parfois d'asymétrique en raison de l'existence de faits et éléments nettement disparates entre certaines Communautés, ne s'est pas réalisée et a plutôt cheminé en sens contraire. Le processus de réforme mené de 2003 à 2006 du Statut de la Catalogne règle de base inștitutionnelle interne mais aussi encadrement juridique des relations avec l'État central - symbolise l'ultime tentative d'amélioration du modèle vers le sens espéré.

L'instrumentalisation politique du processus en question par divers acteurs politiques, sociaux et des médias atteint son degré maximal d'intensité avec une Sentence du Tribunal Constitutionnel, rendue en juin 2010, qui déçoit les aspirations majoritaires du peuple de C atalogne, qui avait approuvé par référendum ce Statut en juin 2006, enclenchant par là même un progressif processus de désaffection à l'égard de l'État espagnol, qui allait devoir samplifier ces dernières années jusqu à provoquer une rupture politique et psychologique avec le cadre politique institutionnel. Les partis politiques, notamment les plus influents dans le système politique catalan, se voyaient dépassés par leurs propres concitoyens qui, de façon plus ou moins spontanée, ou bien encadrés par de nouveaux mouvements politiques ou civiques, envahissaient la rue lors de manifestations ponctuelles ( en juillet 2010, et en 
septembre 2012 et 2013) ou à l'occasion de démonstrations plus radicales à l'instar du mouvement du 15-M , exprimant l'indignation d'une partie des citoyens par rapport à la situation politique, économique et sociale. Dans la plupart de ces manifestations s'entrecroisaient l'indignation d'être instrumentalisés et la volonté d'être reconnus comme des sujets ayant leur propre opinion capable de s'exprimer. La revendication générale du « droit de décider » s'exprimait progressivement lors des manifestations majoritaires avec des slogans en faveur de l'indépendance. On passait ainsi et en peu de temps del'autonomisme et de sa possible amélioration à l'indépendantisme, celui-ci commesentiment et comme volontéd'être d'une majoritédu peuple catalan.

Tout s'est ensuite accéléré au cours des trois ou quatre dernières années bien que le processus de progressive désaffection de la Catalogne et de ses ressortissants vis-à-vis de l'État espagnol ait déjà démarré lors des premières années du $x \mathrm{xl}^{\mathrm{e}}$ siècle.

La dernière décennie 2004-2014 a été marquée par l'échec subi quand il s'est agi de redéfinir la structure territoriale de l'Espagne - lors des discussions et de l'accord sur les nouveaux Statuts d'Autonomie dans diverses Communautés et, en particulier, à partir de la confrontation politique soulevée par le Partido Popular durant le débat sur le nouveau Statut de la Catalogneen 2006 - , par la crise économique que le G ouvernement socialiste de Rodríguez Zapatero tarde à admettre et à affronter jusqu'au milieu de l'année 2010, et par la crise politique et institutionnelle qui a suivi. Cette triple crise qui se reflète en partie également dans l'espace européen dans lequel l'Espagne est intégrée, a conditionné le rôle et l'orientation des divers acteurs politiques, aussi bien ceux officiant en milieu catalan que ceux qui déploient leurs activités sur l'ensemble de l'État espagnol.

À l'occasion du débat politiquequi se déroul equant au modèle degouvernement multi-niveau s'entrecroisent toutes les questions soulevées. La crise économique et sa conséquence politico-institutionnelle placent le débat au niveau étatique et influent sur le cheminement économique et politique du projet européen, en particulier dans ce quel'on appellel'Eurozone. Au niveau de la C atal ogne, on passeégalement d'un débat politique focalisé depuis 2008 sur les mesures à adopter pour surmonter la crise économique, les politiques d'austérité et de réduction de la dépense publique, imposées en grande partie par l'État espagnol lui-même ou par les décisions et initiatives émanant de l'Union européenne, à un débat souverainiste qui finit pratiquement par monopoliser la vie politique catalane.

La fédération elle-même de Convergència i U nió, qui était parvenueà récupérer le G ouvernement de la $\mathrm{G}$ eneralitat de la C atalogne en novembre 2010, avec un projet d'amélioration et de changement du modèle de financement autonomique - grâce au dénommé « Pacte Fiscal », cherchant une proximitéet une assimilation au modèle basque de concertation économique bilatérale - se présente aux élections anticipées de novembre 2012 en se mettant à la tête du mouvement souverainiste qui revendiquele « droit de décider » du peuple catalan. $N$ on sans quelques tensions internes, la fédération deCiU, qui ne parvient pas à affirmer tant dans son programme que dans ses décla- 
rations politiques son engagement en faveur d'une $C$ atalogne indépendante de l'Espagne, entre en étroite compétition politico-électorale avec Esquerra Republica de Cataluña, parti qui se proclame ouvertement indépendantiste mais qui, pour autant, signera un accord de gouvernement avec CiU après les élections de 2012, le regard tourné sur latenued'une consultation ou d'un referendum sur l'indépendance de la $\mathrm{C}$ atalogne dans un délai maximum de deuxans.

Conditionné et parfois stimulé par l'activisme politique et social d'associations récentes, mais au parcours rapide commel'AssembléeN ationaleC atalane (ANC $)^{1}$, comme également l'Association des M unicipalités pour l'Indépendance ${ }^{2}$ ou bien encorele « $\mathrm{C}$ entre Català de N egocis» (CCN $)^{3}$, le Gouvernement de la $C$ atalogne présidé par Artur $M$ as et la majorité parlementaire qui le soutient sesont mis d'accord sur lademande, très majoritairesuivant quasiment tous les sondages, de la reconnaissance du droit de décider et la tenue d'une consultation ou d'un referendum auprès des citoyens de la Catalogne. A travers des appels continusau dialogueavec le G ouvernement espagnol, mais sans attendre ni avancées, ni résultats positifs de ce dialogue qui ne parvient jamaisà s'établir formellement, le G ouvernement de la G eneralitat de la Catalognea mis en place un Conseil Consultatif pour la Transition Nationale auquel il a confié l'élaboration de propositions aussi bien concernant la tenue éventuelle de la consultation référendairequeles diversstadesnécessairesàl'établissement d'un futur État catalan. Il consacre également du temps et des efforts politiques pour transmettreauxinstitutions et chancelleries européennes ses prétentions et propositions dans le but d'internationaliser le conflit soulevé entre la Catalogne et l'État espagnol. Et enfin, sans l'accord du Gouvernement espagnol, mais avec celui d'ERC, ICV-EU iA et les CUP, il vient dedécider dela datedu 9 novembre 2014 pour la tenue de la consultation, du contenu de la question à poser aux citoyens de la Catalogne ainsi que de l'instrument juridique officiel susceptible de rendre possible le déroulement d'un referendum. 0 n dirait que la pression exercée par la base et les inévitables cal culs électoraux ont conduit les dirigeants catalans à une fuite en avant, faisant écho à la vieille affirmation attribuée à De Gaulle: «L'intendance suivra».

Les autres partis catalans, ou bien se situent dans une franche opposition au processus, comme c'est le cas pour le Partido Popular Catalán ou le Ciutadans-partido por la ciudadanía, en dénonçant non seulement l' actuel amalgame de l'expression générale « le droit de décider » avec l'option en faveur d'une C atalogne indépendante de l'Espagne mais égal ement la politique du fait accompli menée par le Gouvernement catalan ; ou bien naviguent entre un vague soutien au « droit à décider » - à condition qu'il s'exerce de ma-

\footnotetext{
1. Association citoyenne créée au début de 2012, rassemblant plus de 25000 associés, qui alancé et organisé les manifestations importantes nettement indépendantistes de septembre 2012 et 2013 et qui conserve une influence importante de proposition politique sur les partis souverainistes.

2. Elle regroupe 685 des 947 communes de C atalogne, mêmesi la seule des capitales catalanesy figurant est Gérone et aucune des grandes communes situées dans la conurbation barcelonaise. 3. Association d'entrepreneurs, de cadres dirigeants et de professionnels favorables à l'indépendance, très influente sur le patronat des PME.
} 
nière légale et en accord avec l'État espagnol - et la proposition d'une alternative fédéraliste pour solutionner le problème de l'insertion de la $C$ atalogne en Espagne; ;'est la position du Parti Socialiste de Catalogne, confronté ces temps derniers à diverses crises internes, en raison de l'ambigüité de ses positions, aggravées à leur tour par l'enlisement du socialisme espagnol et par la crise plus globale de la social-démocratie européenne.

De son côté, la réaction du Gouvernement et des partis politiques nationaux aux positions adoptées en Catalogne ne contribuent pas non plus à régler le problème, ni à apaiser le différend. Le Gouvernement du PP et son Président Mariano Rajoy lui-même passent de l'indifférence à l'opposition frontale à la demande catalane sans exprimer la moindre volonté réelle de dialogue ou d'offre alternative. Dans une position de complète opposition à l'encontre des démarches de la C atalogne, on trouveégalement le parti U nión Progreso y D emocracia (U PyD) au contour résolument nationaliste espagnol. Le PSOE, pour sa part, en admettant l'existence et la gravité du problème politique que représente pour l'Espagne la revendication catalane, offre une alternative générique de réforme fédérale de la Constitution - en phase en l'espèce avec ses homologues du PSC - maisil se situe, par contre, au côté du Gouvernement et du PP dans son hostilité au déroulement d'un référendum en Catalogne. Seule Izquierda U nida, parmi les principaux partis nationaux, fait montre de compréhension face aux initiatives catalanes et se prononce en faveur dela tenue de la consultation en $C$ atalogne.

L'action du Gouvernement du PP, tirant argument des mesures indispensables pour affronter la crise financière espagnole et la crise économique plus généralement, ne cache pas son intention de lancer un signal fort de recentralisation, qui s'accompagne de l'inertie ou du dédain à l'égard des attentes en provenance de la C atalogne. D e son côté, le Gouvernement catalan et la majorité parlementaire qui appuie le processus souverainisteet, en comptant en beaucoup d'occasions sur le soutien du PSC et de ICV-EU iA, interprètent n'importe quelle mesure du Gouvernement espagnol comme un affront permanent ou un laminage du gouvernement autonomiquecatalan et une forme deméprisà l'égard d'une réalité nationale, historique, économique, culturelle ou linguistique.

À la vue de cette confrontation politique, ce que l'on a fini par dénommer « $e$ choque de trenes » paraît inévitable a moins que les deux parties soient disposées à amorcer un dialogue qui évite la polarisation entre les deux extrêmes : la recentralisation progressive et l'inversion du processus autonomique qui semble régir une large part de l'action du Gouvernement espagnol, faceà l'eventuelle sécession de la C atalogne del'Espagne interprétée par leurs défenseurs comme la solution à tous les maux frappant la Catalogne, aussi bien économiques que politiques.

Dans l'opinion publique catalane et espagnole, certaines voix se sont levées pour réclamer une ouverture au dialogue de la part du Gouvernement espagnol qui demeure strictement cramponné aux limites établies par la C onstitution. U n dialoguequi permettrait un remodelage du modèle territorial acceptant des croissances asymétriques. Et qui lancerait en même temps 
un appel à la prudence de la part du Gouvernement catalan face à l'accélération demandée par les associations favorables à une issue rapide et résolue vers l'indépendance. $0 \mathrm{n}$ demande davantage d'intelligence émotionnelle dans ce débat politique. Si une part très importante des forces politiques catalanes, de leurs organisations syndicales et, en particulier de leur opinion publique - ainsi que l'attestent aussi bien les enquêtes que les mobilisations citoyennes - demandent encore àêtre écoutées, il ne semble pasjudicieux de refuser la quête d'une issue raisonnable démocratiquement parlant. D 'autant plus que pratiquement latotalitédel'opinion académique constitutionnaliste catalane et, une large partie même de l'espagnole, affirme qu'une consultation comme celle qui est demandée en $C$ atalogne s'inscrit parfaitement dans le cadre constitutionnel actuel. Peut-être peut-on objecter que la Constitution espagnole n'envisage pas, comme d'ailleurs c'est le cas dans l'immense majorité des pays démocratiques, le droit à la sécession d'une partie de son territoire mais sur un plan strictement démocratique il paraît très difficile de fermer lesyeux et de se boucher les oreilles devant la réclamation et la volonté d'être écoutés, clairement exprimées par les citoyens de C atalogne, dans leur très grande majorité.

En même temps, à l'instar de ce qui est en train de se produire en Écosse - qui logiquement semble une référence pour la $C$ atalogne et un modèle à suivre, en raison de l'accord conclu entre le Gouvernement écossais et le Gouvernement britanniquepour latenue d'un referendum sur son éventuelle indépendance par rapport au Royaume-U ni - une partie du débat tourne autour de l'appartenance de la C atalogne à l'U nion européenne dans I'hypothèse de son eventuel divorce avec l'État espagnol. Du milieu académique et du monde politique émanent opinions et affirmations en tous sens : depuis la conviction pleine et entière que le fait de continuer à demeurer au sein de I'U nion est possible jusqu'au refus de toute possibilité de pouvoir s'y maintenir. Au sein du Gouvernement espagnol, ce thème de discussion, mais également certains autres concernant plutôt la viabilité politique et économique d'une Catalogne indépendante, ont été abordés en priorité par le M inistre des Affaires étrangères qui apparait ainsi curieusement comme le principal interlocuteur espagnol dans la confrontation dialectique C atalogne-Espagne.

Par ailleurs, la question de la place au sein de l'U nion européenne d'une éventuelle Catalogne indépendante ainsi que la démarche des catalans afin que les autorités communautaires infléchissent les positions du Gouvernement espagnol arcboutédans son refus de dialoguer et de chercher un accord avec le G ouvernement catalan, ont conduit divers dirigeants européensàseprononcer, quecesoitlePrésident delaCommission ou celui du ParlementEuropéen mais également divers commissaires ou certains mandataires des pays de l'U nion. Tous ont manifesté des choix différents, les uns adoptant une position distante en considérantqu'il s'agit làd'unequestion intérieurequi concerneuniquement les autorités espagnoles et catalanes, les autres, exprimant des considérations plus ou moins radicales en regard des difficultés juridiques et politiques soulevées par la pérennité de la place de la Catalogne au sein del'U nion européenne ou son integration en tant que nouvel État indépendant. 
Dans un tel contexte il parait évident que l'absence de dial ogue politique entre G ouvernements et forces politiques et l'impossibilité qui s'en suit d'arracher un accord offrant une issue aux démarches apparues comme majoritaires en Catalogne, risque de déboucher sur une escalade dans la confrontation, sur une situation confuse de déroulement d'un referendum même pas toléré par le Gouvernement espagnol, sur de nouvelles élections autonomiques catalanes anticipées - dont l'objectif majeur, quasi unique du débat politico-électoral entre les forces politiques en concurrence reposerait sur la future indépendance de la Catalogne - et, en fonction de ses résultats, sur une éventuelle déclaration unilatérale d'indépendance de la part de la nouvelle majorité parlementaire que tous les sondages accordent actuellement à l'alliance ERC et CiU, encore qu'elle puisse compter égal ement sur l'appui d'autres forces minoritaires.

Ce scénario hypothétique mais possible, se heurte à diverses considérations politiques et opinions académiques analysant les conséquences que pourrait avoir alors la sécession de la C atalogne par rapport à l'État espagnol, non seulement pour la $\mathrm{C}$ atalogne elle-même maiségal ement pour l'E spagne, pour l'Eurozone et pour l'ensemble de l'U nion européenne. Si, comme le soulèvent divers experts économiques l'Espagne a été considérée à maintes reprises par le FM I et, dans une certaine mesure par les responsables économiques de I'U E, comme un danger systémique pour l'avenir de la stabilité financière de l'euro, l'hypothèse d'une Catalogne indépendante soulève, à court et à moyen termes, bien des problèmes aux citoyens catalans et, sans nul doute, du reste de l'Espagne et de l'Europe. M ais c'est aussi en raison des conséquences politiques d'une rupture sans aucun accord d'un État de l'Union, élément qui différencie également la situation catalane du cas écossais.

Parmi ces problèmes, celui de lanature del'indépendance du pays ( plutôt que de sa légitimité constitutionnelle) pourrait faire l'objet d'un débat. Certains théoriciens du Fédéralisme considèrent que les résultats irréversibles de l'intégration européenne permettent de penser la venue d'un nouveau type de société en Europe ; celui d'un Fédéralisme sans État. T rès proche d'une évolution àla C anadienne, ce régime serait celui d'uneépoque post-nationale où ce sont des structures et des procédures de négociation des intérêts et des conflits qui l'emporteraient sur les formes régaliennes du pouvoir que l'Europe a inventé et exporté depuis le traité deW estphalie. En d'autres termes le système actuellement existant du pouvoir dans l'U nion européenne (qui est tout sauf un État) pourrait être reproduit dans son espace en transition. Cela présuppose une émancipation de l'héritage de la souveraineté incarnée par I'État sous quelque forme que ce soit, même fédérale à l'Allemande. Autrement dit, l'entrée dans ce cycle de fédéralisme post-étatique ouvrirait la voie à un nouvel âge autogestionnaire, tout au moins à une promotion de formes diverses de démocratie participative tant sociale que politique.

Pourquoi la Catalogne ne serait-elle pas le champ de ce tournant historique? D ans la période de la sortie de la dictature, elle a soulevé bien des espoirs d'un nouvel « État des autonomies », l'Espagne semblant capable 
d'installer un pouvoir fragmenté par autant deterritoireséconomiques, culturels et politiques qu'elle reconnaissait de statuts spécifiques à ses « régions ». Et beaucoup ont pu espérer voir ainsi s'inventer une nouvelleformed'organisation du pouvoir dépassant l'unité et la centralité plus ou moins au principe de chacun des États-membres de I'U nion européenne. À réclamer comme aujourd'hui l'indépendance, la Catalogne pourrait relancer cette perspective en s'appuyant sur ce qu'est devenue l'U nion réellement existante, mais en faisant son deuil d'une reconnaissance comme État. Ce sacrifice pourrait s'avérer alors terriblement attractif pour tous les peuples d'Europe qui n'en finissent pas de creuser leurs identités particulières et parfois leurs tombes.

Lesarticles qui constituent cenuméro thématique dePôleSud souhaitent projeter un éclairage sur la situation traversée par la C atalogne ces temps derniers, dans un cadre européen aussi complexe face à de nouveaux défis, et ceci en se penchant sur divers aspects de la vie sociale, politique et culturelle catalane.

Le premier article est consacré à l'analyse, du point de vue historique, de l'essor du nationalisme catalan de la fin du XIX ${ }^{e}$ sièclejusqu'ànosjours. Ensuite on aborde les mutations assumées par la société catalane au cours de plus de trente années d'autonomie politique recouvrée, à savoir de 1980 à nos jours. D eux contributions sont consacrées à l'analyse de la politique catalane actuelle, l'une au prisme des politiques publiques menées aussi bien dansl'exercice de son autogouvernement ( " self rule») quedans sa capacité de gouvernement partagé( « shared rule») dans un système multi-niveau comme celui dans lequel s'intègre la C atalogne; et l'autre, en focalisant sur l'analyse des relations politiques, économiques et fiscales entre Catalogne et Espagne, en abordant l'évolution récente du système de financement des Communautés et, en particulier, la Catalogne. D eux autres articles se consacrent à la description et à l'analyse de la vie politique intérieure de la C atalogne, en étudiant sa trajectoire au gré des diverses compétition sélectorales, les prévisions que les études actuelles apportent au débat sur l'indépendance de la Catal ogne ainsi que la constitution et l'évolution de son propre système de partis. Le décryptage de la réalité culturelle de la C atalogne est opéré dans un premier article, plus descriptif et analytique de sa complexité et de ses contradictions, tandis queles perspectives de continuité et de changement des politiques publiques culturelles font l'objet d'un second article. Enfin, on aborde l'intense activité extérieure déployée par la Catalogne et ses institutions de gouvernement autonomique, aussi bien quand il s'agit de celle qui est considérée comme normale, en accord ou tolérée par la G ouvernement espagnol que celle qu'on suppose être, en particulier dans le contexte du débat politique actuel, objet d'affrontement avec le Gouvernement de l'Espagne. 